\title{
FORMAL CONCEPT ANALYSIS IN DESIGN
}

\author{
MIHALY LENART \\ University of Kassel, Germany
}

\begin{abstract}
Formal concept analysis is a research method using set theoretical models of concepts and their hierarchical orderings. The model is based on concept or Galois lattices whose application for analyzing design contexts has been proposed earlier by Ho (1982a). Although concept lattices have been widely used for analyzing contexts in various areas, such as music, social sciences, or cognitive science, the analysis of design contexts by concept lattices has not gained acceptance. In particular, it has not been recognized yet that Hasse diagrams, a representation of concept lattices by line diagrams, can not only help to visualize important hidden properties of a design context, but also provide a new kind of analytical tool that can support the decision making process. The paper shows that Hasse diagrams contain all information of the underlying context and reveal inherent structural dependencies not captured by any other graphical representation of the given context. It shows also how concept lattices, or rather their Hasse diagrams, can be used for supporting the design process in general and for analyzing design contexts in particular.
\end{abstract}

\section{Introduction}

Many attempts have been made to describe design contexts exactly by formal, mathematical means. The purpose of such description is to understand relations or dependencies between design objects and to develop computer programs for automating certain parts of the design process. One way to characterize a design context is to describe design objects as elements of a set and relations and/or operations on these elements. The result is an algebraic model of the design context. There are numerous such models and the formal description of a context we are using here is probably the simplest one. Nevertheless, this simple model can capture basic features of a design context. The same model has been used also for Q-analysis that was developed by Atkin (1974) in the early 1970s. It was quite popular and widely used for the analysis of design contexts in the late 1970s and early 1980s. It turned out, however, that some of the information get lost in the process and other algebraic tools, such as concept lattices, are superior to or more adequate than Qanalysis for analyzing design concepts. 
In the early 1980 s, Ho has proposed the use of new algebraic tools, in particular set and category theoretical ones, for a formal description of the design process (Ho, 1982a; Ho, 1982b; Ho, 1982c). One of these tools was concept or - as Ho called them - Galois lattices. Although Ho's proposal was novel to design, concept lattices have been widely used previously in other social and scientific areas. The ground work for these applications has been laid down by Wille (1981; $1983 ; 1984 ; 1987 ; 1992)$. He has developed not only the lattice theoretical foundations but also various lattice generation and representation methods. Beyond the theoretical part, Wille and his co-workers have demonstrated the scope and the power of these methods by numerous applications. The aim of this paper is to utilize Wille's methods for design purposes.

\section{Concept Lattices}

Concept analysis is based on a common data type called (formal) context. From a context several mathematical objects, such as concept lattices, can be derived.

Definition 2.1 $A$ context is a triple $(O, P, \gamma)$ where $O$ and $P$ are sets and $\gamma \subseteq$ $O \times P$ is a binary relation between $O$ and $P$.

The elements of $O$ and $P$ describe entities of a given design situation or problem. According to March (1982), we need at least "two descriptive systems" in order to be able to describe design tasks or processes. In many cases two distinctive systems are also sufficient to describe relevant characteristics of a design context. A simple example is given in Figure 1 describing building structures and their main characteristics.

The two sets $O$ and $P$ can be chosen arbitrarily, however, for all practical purposes the "two distinctive description systems" mean that we usually have two sets of different kinds of entities, such as facts and values, objects and features, forms and functions, or locations and activities (Ho, 1982a). Keeping this in mind, we can assume - without loss of generality - that $O$ is a set of objects and $P$ is a set of properties. If an object $o \in O$ has a property $p \in P$, i.e. $(o, p) \in \gamma$ then we write $o \gamma p$. The context $(O, P, \gamma)$ can be represented by a matrix with the objects heading the rows and the properties the columns. If an object $o_{i}$ has the property $p_{j}$ then we put an $\mathrm{x}$ into the field $(i, j)$ and leave it empty otherwise (see Figure 1). For all subsets $X \subseteq O$ and $Y \subseteq P$ we define the following derivation operations represented by "prime":

$$
\begin{aligned}
& X \longmapsto X^{\prime}=\{p \in P \mid \text { orp forall } o \in X\} \\
& Y \longmapsto Y^{\prime}=\{o \in O \mid \text { orp forall } p \in Y\}
\end{aligned}
$$

Let $\wp X$ denote the power set of $X$ and $\wp Y$ the power set of $Y$. Then the pair $(F, G)$ of mappings $F: X \wp X^{\prime}$ and $G: Y \wp Y^{\prime}$ are said to form a Galois connection between $\wp X$ and $\wp Y$ for which the following properties hold (Birkhoff, 1967):

$$
X_{1} \subseteq X_{2} \text { implies } X_{2}^{\prime} \subseteq X_{1}^{\prime} \text { for } X_{1}, X_{2} \subseteq O
$$




\begin{tabular}{|c|c|c|c|c|c|c|}
\hline building structure & 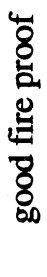 & 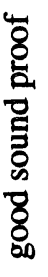 & 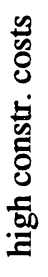 & 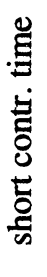 & 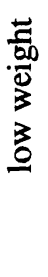 & $\begin{array}{l}\text { ज्ञ } \\
\text { की } \\
\text { 60 } \\
\text { 으 }\end{array}$ \\
\hline cast in place & $\mathbf{x}$ & $\mathbf{x}$ & & & & $\mathbf{x}$ \\
\hline steel frame & & & $\mathbf{x}$ & $\mathbf{x}$ & $\mathbf{X}$ & $\mathbf{x}$ \\
\hline wood frame & & & & $\mathbf{x}$ & $\mathbf{x}$ & \\
\hline aluminum frame & & & $\mathbf{x}$ & $\mathbf{x}$ & $\mathbf{x}$ & \\
\hline masonry & $\mathbf{x}$ & $\mathbf{x}$ & & & & \\
\hline prefab. concrete & $\mathbf{X}$ & $\mathbf{x}$ & & $\mathbf{x}$ & & $\mathbf{x}$ \\
\hline
\end{tabular}

Figure 1. A context describing relations between building structures and structural properties.

$$
\begin{array}{r}
Y_{1} \subseteq Y_{2} \text { implies } Y_{2}^{\prime} \subseteq Y_{1}^{\prime} \text { for } Y_{1}, Y_{2} \subseteq P \\
X \subseteq X^{\prime \prime} \text { and } X \subseteq X^{\prime \prime \prime} \text { for } X \subseteq O \\
Y \subseteq Y^{\prime \prime} \text { and } Y \subseteq Y^{\prime \prime \prime} \text { for } Y \subseteq P \\
\left(\bigcup_{t \in T} X_{t}\right)^{\prime}=\bigcap_{t \in T} X_{t}^{\prime} \text { for } X_{t} \subseteq O(t \in T) \\
\left(\bigcup_{t \in T} Y_{t}\right)^{\prime}=\bigcap_{t \in T} Y_{t}^{\prime} \text { for } Y_{t} \subseteq P(t \in T)
\end{array}
$$

The relations above show a natural 'duality' between objects and properties.

Definition 2.2 For a given context $(O, P, \gamma)$, we call a pair $(A, B)$ a concept if $A \subseteq O, B \subseteq P, A=B^{\prime}$ and $B=A^{\prime}$. A and $B$ are called the extent and the intent of the concept $(A, B)$ respectively.

Figure 2 shows all the 11 concepts of the context of Figure 1.

Let us denote the set of all concepts of the context $(O, P, \gamma)$ by $\mathbf{L}(O, P, \gamma)$. It can be shown that there is an ordering structure on $\mathrm{L}(O, P, \gamma)$ defined by the set inclusion relation: For any two concepts $\left(A_{1}, B_{1}\right)$ and $\left(A_{2}, B_{2}\right)$ we have $\left(A_{1}, B_{1}\right) \leq$ $\left(A_{2}, B_{2}\right)$ if and only if $A_{1} \subseteq A_{2}$ which is equivalent to $B_{2} \subseteq B_{1}$ by 4 . In this case we call $\left(A_{1}, B_{1}\right)$ a subconcept of $\left(A_{2}, B_{2}\right)$ and $\left(A_{2}, B_{2}\right)$ a superconcept of $\left(A_{1}, B_{1}\right)$. For the subconcept-superconcept relation we denote the ordered set $(\mathrm{L}$ $(O, P, \gamma), \leq)$ by $\underline{\mathbf{L}}(O, P, \gamma)$. 


\begin{tabular}{|l||l|l|}
\multicolumn{2}{|c|}{} & \multicolumn{1}{c|}{} \\
\hline 1 & 0 & $\mathrm{~A}^{\prime}=\mathrm{B}$ \\
2 & $\mathrm{~B}$ & $1,2,3,4,5,6$ \\
3 & $\mathrm{~b}$ & $3,4,5,6$ \\
4 & $\mathrm{f}$ & $1,2,4,6$ \\
5 & $\mathrm{a}, \mathrm{f}$ & $1,2,6$ \\
6 & $\mathrm{~b}, \mathrm{~d}$ & $3,4,5$ \\
7 & $\mathrm{~b}, \mathrm{f}$ & 4,6 \\
8 & a,b,f & 6 \\
9 & a,e,f & 1,2 \\
10 & b,c,d & 4,5 \\
11 & b,c,d,f & 4 \\
\hline
\end{tabular}

Figure 2. The concepts derived from the building structure context.

In order to formulate the basic theorem showing that $\underline{\mathrm{L}}(O, P, \gamma)$ is a complete lattice, we need to introduce some lattice theoretical notations ${ }^{1}$.

Definition 2.3 A partially ordered set $P$ (or for short poset) is a set with a binary relation $\leq{ }^{2}$ such that for all $x, y, z \in P$ we have reflexivity: $x \leq x$, antisymmetry: if $x \leq y$ and $y \leq x$ then $x=y$, and transitivity: if $x \leq y$ and $y \leq z$ then $x \leq z$

Such a (reflexive, symmetric and transitive) relation is called a partial order on $P$. If any two elements of a poset are $\leq-$ related we say that the poset is a totally ordered set or a chain.

Definition 2.4 If $X$ is a subset of a poset $P$ (i.e. $X \subseteq P$ ), then $a \in P$ is an upper bound of $X$ if for each element $x \in X a \geq x$. The least upperbound or supremum of $X$ denoted by sup $X(\vee X)$ is the smallest upperbound of $X$. (It can easily be shown that each subset has at most one.) Similarly, an element $b \in P$ is a lower bound of a subset $X \subseteq P$ iffor each $x \in X b \leq x$. The greatest lower bound or infimum of $X$ denoted by inf $X(\wedge X)$ is the greatest of all lower bound of $X$.

Definition 2.5 A lattice $\mathcal{L}$ is a poset $P$ such that any two elements $x, y \in P$ have a supremum called 'join' and an infimum called 'meet'. Therefore join and meet are (total, binary) operations on a poset $P$ and for any two elements $x$ and $y$ of a lattice we write $x \vee y$ for their join and $x \wedge y$ for their meet.

\footnotetext{
${ }^{1}$ For a detailed discussion see Birkhoff (1967)

${ }^{2}$ Notice that $\leq$ is used here in a more general sense than in the case of concepts.
} 
Definition 2.6 A lattice $\mathcal{L}$ is called complete if each subset $X \subseteq P$ has a supremum and an infimum in $\mathcal{L}$. We also say that $\mathcal{L}$ is closed under supremum and infimum.

Since we restricted ourselves to finite examples, all our lattices will be also finite. Since any finite lattice is complete, we can take completeness of our lattices for granted.

Definition 2.7 A subset $D$ of a complete lattice $\mathcal{L}$ is called infimum dense if each element of the a lattice is an infimum of a subset $X$ of D i.e. $\mathcal{L}=\{\wedge X \mid X \subseteq D\}$. Similarly a subset of a complete lattice $\mathcal{L}$ is called supremum dense if $\mathcal{L}=\{\vee X \mid$ $X \subseteq D\}$.

Now we can state the basic theorem mentioned earlier (Wille, 1992):

Theorem $2.1 \underline{\mathbf{L}}(O, P, \gamma)$ is a complete lattice of the context $(O, P, \gamma)$ whose infimum is:

$$
\bigwedge_{t \in T}\left(A_{t}, B_{t}\right)=\left(\bigcap_{t \in T} A_{t},\left(\bigcup_{t \in T} B_{t}\right)^{\prime \prime}\right)
$$

and supremum is:

$$
\bigvee_{t \in T}\left(A_{t}, B_{t}\right)=\left(\left(\bigcup_{t \in T} A_{t}\right)^{\prime \prime}, \bigcap_{t \in T} B_{t}\right.
$$

Conversely, a complete lattice $\mathcal{L}$ is isomorphic to $\underline{\mathrm{L}}(O, P, \gamma)$ if and only if there are mappings $\sigma: O \longrightarrow \mathcal{L}$ and $\varrho: P \longrightarrow \mathcal{L}$ such that $\sigma O$ is supremum-dense in $\mathcal{L}$ and $\varrho P$ is infimum-dense in $\mathcal{L}$, and o $\gamma p$ is equivalent to $\sigma o \leq \varrho p$ for all $o \in O$ and $p \in P$, i.e. $\mathcal{L} \cong \underline{\mathbf{L}}(\mathcal{L}, \mathcal{L}, \leq)$ and, if $\mathcal{L}$ has a finite length, $\mathcal{L} \cong \underline{\mathbf{L}}(\mathcal{J}(\mathcal{L}), \mathcal{M}(\mathcal{L}), \leq)$. We call $\underline{\mathbf{L}}(O, P, \gamma)$ a concept lattice.

\section{Generating and Drawing Concept Lattices}

Before going into the discussion of how to interpret information provided by concept lattices or rather how to use concept lattices for design purposes, let us discuss the following questions: How can we obtain the concept lattice of a given context, and how can the concept lattice be represented conveniently by easily surveyable line diagrams? In fact, the primary aim of this paper is to discuss the use of such diagrams as decision support tools in design rather than discussing theoretical questions related to concept lattices.

The most obvious derivation method to obtain all concepts from a context $(O$, $P, \gamma)$ is to generate for all subsets $X \subseteq O$ the concept $\left(X^{\prime \prime}, X^{\prime}\right)$ by 5 or for all subsets $Y \subseteq P$ the concept $\left(Y^{\prime}, Y^{\prime \prime}\right)$ by 6 By ordering the concepts using the $\leq-$ relation, we obtain the concept lattice $\underline{\mathbf{L}}(O, P, \gamma)$. Even though this method might work well for smaller contexts, it requires the generation of at least $2^{|O|}$ subsets and their "prime" derivates, if $O \leq P$ or at least $2^{|P|}$ subsets if $P<O$. (It is also possible to use a combination of subsets $X$ and $Y$ in order to obtain all concepts.) In case of larger contexts, this method becomes obviously inefficient. It is more 


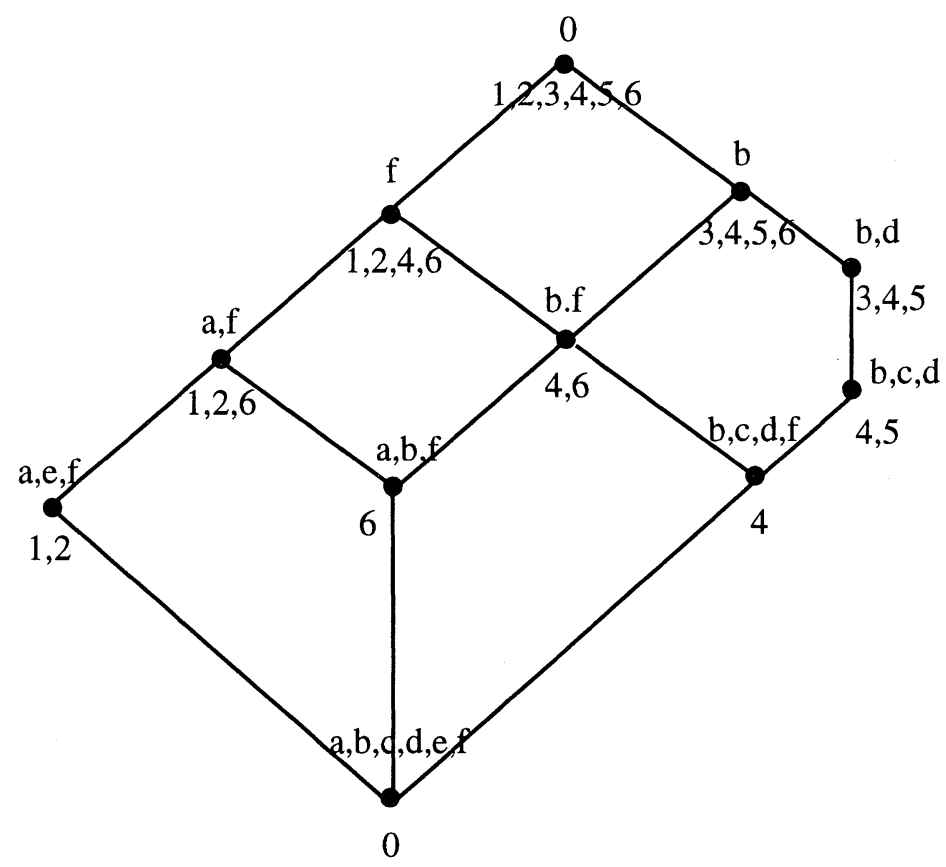

Figure 3. Hasse diagram of the concept lattice generated from the building structure context.

efficient to use the formulae $X^{\prime}=\bigcap_{o \in X}\{o\}^{\prime}$ or $Y^{\prime}=\bigcap_{p \in Y}\{p\}^{\prime}$ which are special cases of 7 and 8 , and then form $\left(X^{\prime \prime}, X^{\prime}\right)$ or $\left(Y^{\prime}, Y^{\prime \prime}\right)$. This means that we can start with arbitrary intents $\{o\}^{\prime}(o \in O)$ or extents $\{p\}^{\prime}(p \in P)$ and derive new extents and intents by the above formulae. Repeating the process provides all concepts, since every extent or intent is the intersection of certain other extents or intents. There are other, more efficient ways to generate the concepts by computer as well. For a discussion and the comparison of concept generating algorithms see Gantner (1987).

Lattices, in particular concept lattices, are usually represented by line diagrams, called Hasse diagrams. The nodes of the diagrams represent concepts and if we have $\left(A_{1}, B_{1}\right) \geq\left(A_{2}, B_{2}\right)$ then we place the node representing $\left(A_{1}, B_{1}\right)$ higher in the diagram than the node representing $\left(A_{2}, B_{2}\right)$. If $\left(A_{1}, B_{1}\right) \geq\left(A_{2}, B_{2}\right)$ and there is no concept $\left(A_{3}, B_{3}\right)$ such that $\left(A_{1}, B_{1}\right) \geq\left(A_{3}, B_{3}\right) \geq\left(A_{2}, B_{2}\right)$ then the nodes representing $\left(A_{1}, B_{1}\right)$ and $\left(A_{2}, B_{2}\right)$ are connected by a line in the diagram. This representation of the concept lattice is a graph displaying the ordering relation. Such a graph is called Hasse diagram. Figure 3 shows the Hasse diagram of the concept lattice generated from the context of Figure 1. In the diagram the intent is placed above and the extent below the corresponding node.

It is, however, sufficient to attach the name of an object $o$ to a node representing $\sigma o:=\left(\{o\}^{\prime \prime},\{o\}^{\prime}\right)$ which is the smallest concept containing $o$ in its extent, and 


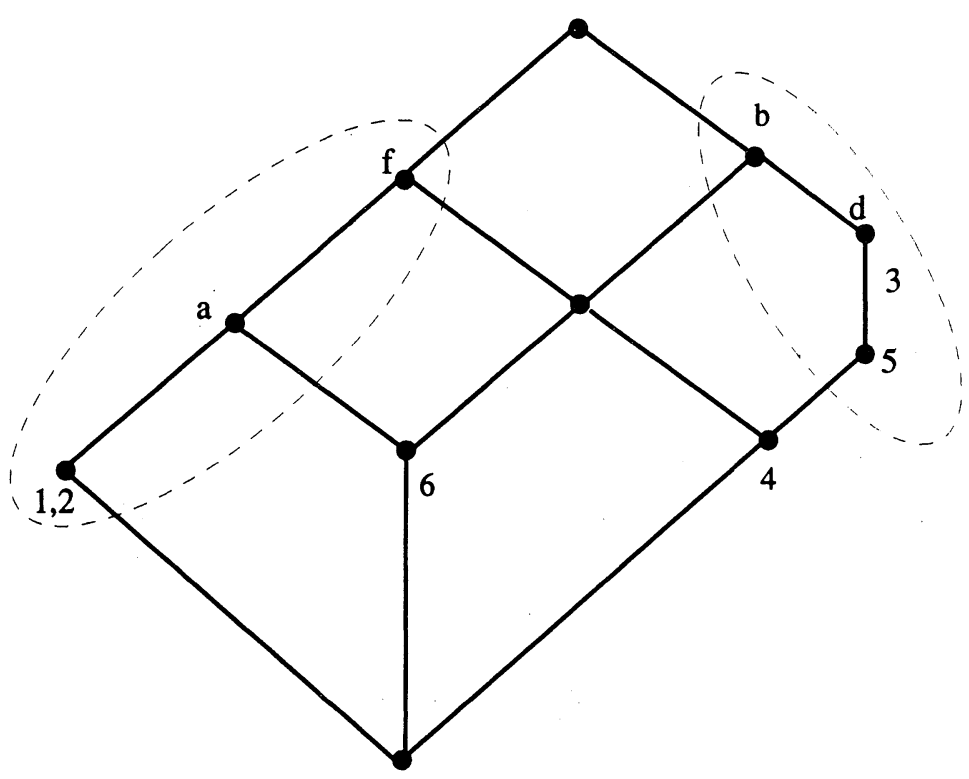

Figure 4. The simplified Hasse diagram of the building structure lattice.

similarly, it is sufficient to attach the name of a property to the node representing $\varrho p:=\left(\{p\}^{\prime},\{p\}^{\prime \prime}\right)$ which is the largest concept containing $p$ in its intent. This follows from $o \in A \Leftrightarrow \sigma o \leq(A, B)$ and $p \in B \Leftrightarrow \varrho p \geq(A, B)$. Figure 4 shows the simplified Hasse diagram. The missing labels can easily be obtained by consecutive addition of extents or intents along the upward or downward sloping paths.

Any concept (or other kinds of) lattice can be represented by an infinite number of different Hasse diagrams. Some diagrams are more, other less appropriate for representing and analyzing lattice properties. Therefore, regardless of the particular use of the lattice, there are some guide lines for generating 'nice' and useful diagrams. The most important one is that the diagram should display or even emphasize important structural lattice properties. This can be achieved by various methods and we will discuss one of these methods in section 5. It is also desirable that connecting lines are straight sloping segments. ${ }^{3}$ It is also common to draw planar or 'as planar as possible' diagrams, i.e., to minimize the number of crossing lines. Hasse diagrams are often composed of parallelograms or rather the underlying grid of the diagram is parallelogram grid. Boolean lattices can nicely be represented by $\mathbf{n}$-dimensional cubes drawn on such grids. This is the reason that Hasse diagrams are often composed of Boolean sublattices depicted as $\mathbf{n}$-dimensional cubes. (For

\footnotetext{
${ }^{3}$ Sometimes, however, we want to represent important features that can be achieved only by abandoning straight lines.
} 


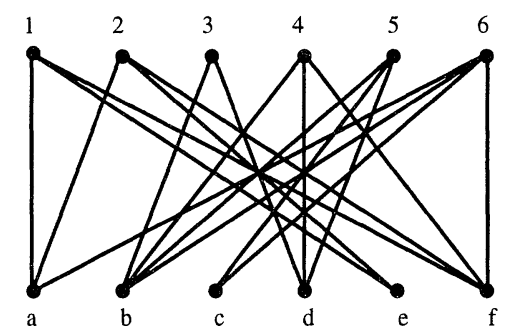

Figure 5. Representation of the building structure context by a bipartite graph.

more on the representation of lattices by line diagrams see Rival (1989).)

Besides concept lattices, other methods can be used for representing structural properties of a context as well. First of all the context might not be given in the (convenient) matrix form as in Figure 1, such that recognizing objects, properties and relations itself can be a representational task. For example, the list $\ell_{P}=$ $(1,2,6 ; 3,4,5,6 ; 4,5 ; 3,4,5 ; 1 ; 2 ; 1,2,4,6)$, or the list $\ell_{O}=(a, e, f ; a, e, f ; b, d ;$ $b, c, d, f ; b, c, d ; a, b, f)$ describes the exact same context as Figure 1 . Although this representation is more efficient and better for programming purposes, it is hard to recognize structural properties by such lists. We can also represent the same context by a bipartite graph as it is shown in Figure 5. This representation is also hard to read and provides little information about the structure of the context.

In contrast, as Wille (1992) states: "Concept lattices constitute a structural analysis of data contexts without reducing the the data. A labeled line diagram of a concept lattice still represents all knowledge 4 coded in the underlying context and, furthermore, unfolds (and reveals to the eye) the inherent conceptual structure of the coded knowledge." In fact, concept lattices display information provided by all other structural representation methods. For example hierarchical concept clustering provides clusters like the one of building structures with good fire and sound proof capabilities, or the one of those with short construction time and low weight (see Figure 6). The same clusters can easily be obtained from the concept lattice in Figure 4 where we can find these two clusters on the left and right branches of the diagram respectively.

The concept lattice also represents all implications between the attributes. An implication $Y \longrightarrow Z$ of a context $(O, P, \gamma)$ is a pair of subsets $Y, Z \subseteq P$ such that $Y^{\prime} \subseteq Z^{\prime}$, i.e., each object of $O$ that has all the properties of $Y$ will also have the properties of $Z$. Similarly, the implication $X \longrightarrow T$ is a pair of subsets $X, T \subseteq O$ such that $X^{\prime} \subseteq T^{\prime}$,i.e., each property of $P$ that belongs to the the objects of $X$ will belong also to the objects of $T$. This can be considered as an 'inheritance'relation in object oriented terms that is displayed by the diagram. We can read the implications from the diagram because of Theorem 2.1, $Y^{\prime} \subseteq Z^{\prime} \Leftrightarrow \forall l \in Z$ :

\footnotetext{
${ }^{4}$ Emphasis added.
} 


\section{short installation}

fire \& sound proof

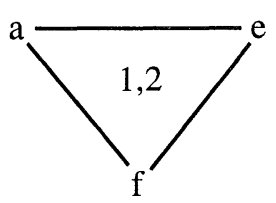

$\&$ low weight

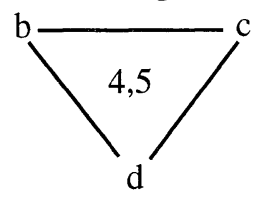

Figure 6. Two clusters whose elements share the same properties.

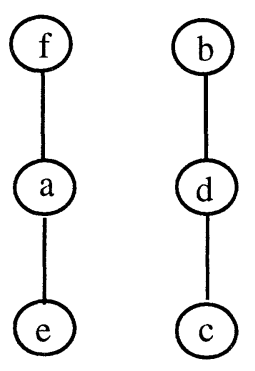

Ordering of objects

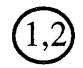

(6)

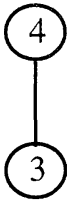

Figure 7. Ordering of the properties and objects.

$\left(Y^{\prime}, Y^{\prime \prime}\right) \leq \varrho l$ in $\underline{\mathrm{L}}(O, P, \gamma)$ and $\left(Y^{\prime}, Y^{\prime \prime}\right)=\bigwedge_{k \in Y} \varrho k$ and $X^{\prime} \subseteq T^{\prime} \Leftrightarrow \forall n \in$ $T:\left(X^{\prime \prime}, X^{\prime}\right) \leq \sigma n$ in $\underline{\mathbf{L}}(O, P, \gamma)$ and $\left(X^{\prime \prime}, X^{\prime}\right)=\bigvee_{m \in X} \sigma m$. For example $\{a, c\} \longrightarrow\{e, f\}$ because $\sigma a \wedge \sigma c \leq \sigma e$ and $\sigma a \wedge \sigma c \leq \sigma f$, i.e., the properties of $a$ and $c$ will also be properties of $e$ and $f$.

Implications with one element premise provide a natural ordering on the set of properties by $k \leq l: \Leftrightarrow l \longrightarrow k(\Leftrightarrow \varrho l \leq \varrho k)$ and similarly, we obtain a natural ordering on the set of objects by $m \leq n: \Leftrightarrow m \longrightarrow n(\Leftrightarrow \sigma m \leq \sigma n)$. This ordering is shown in Figure 7. Figure 8 shows implications on the objects.

\section{Analyzing Contexts by Concept Lattices}

The following example from Lenart (1990) describes a common design task by statements and their connections. (Another example in Lenart (1988) shows another novel application of concept analysis in design.) The extents of the concepts are statements about the current situation at a certain university describing the problem of having unsatisfactory student accommodations. Using Ho's (1982a) terms, these statements represent the state space of the design process. The intents of the concepts are sets of decisions changing the current situation. This corresponds to the decision space (Ho, 1982a). The context is represented as a matrix in Figure 

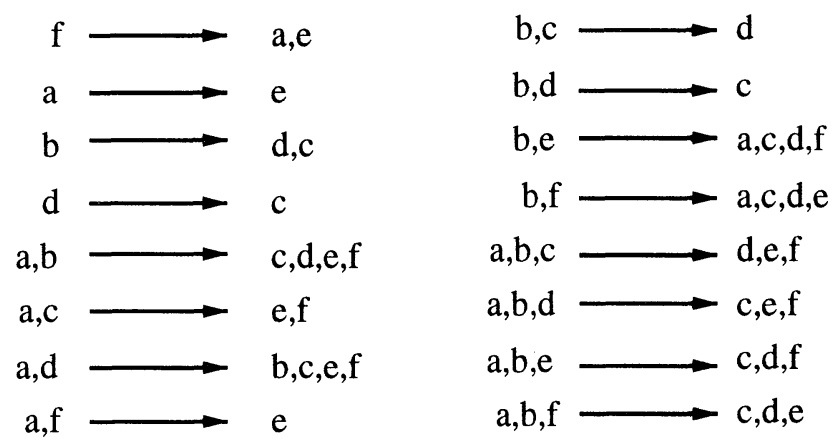

Figure 8. Implications on the objects.

9. In this context $x \gamma y$ means that the statement $x$ is effected by the decision $y$, or in other words, the decision $y$ changes the validity of the statement $x$. We notice that we did not specify how the decision $y$ effects the statement $x$ nor the strong or weakness of the influence. However, by refining the relation between the two sets (i.e. expanding the context) several modifications are possible. The new context replaces a single statement by a certain number of sub statements and defines the relation on these new statements. Such a method for the extension or refinement of contexts was described in Macgill (1983). However, it is also possible to have many values or a scale describing the relationship between objects and properties. A method for dealing with many-valued contexts is described in Wille (1987; 1992).

The problem is obviously simplified by describing it with only a few number of statements using a binary relation whether certain statements are mutually related or not. Although this fictive problem is quite common and realistic, this small example shows that even in such simplified cases the context can be quite complicated, yet concept analysis provides a powerful tool to analyze the problem and to help in the decision making process. Like in the previous example, the concept lattice can be generated by calculating all possible concepts, ordering the concepts, and finally drawing the concept lattice. Even though the context of this simple task is small, it provides 63 concepts and the resulting lattice, as Figure 10 shows, is quite complex.

Obviously, there are infinitely many different drawings representing the same lattice. However, not all the diagrams represent the lattice 'nicely'. A 'nice' drawing means that we can 'read' the diagram, i.e. obtain information (dependencies) easily. One trick we might want to use is to look for Boolean sublattices (or 'almost'Boolean sublattices, as mentioned earlier) and draw them as n-dimensional cubes with parallel edges. The search for Boolean sublattices is not difficult and can systematically be done. However, putting the entire diagram together requires some skill. Because of the difficulties in drawing Hasse-diagrams (especially 'nice' ones) 


\begin{tabular}{|c|c|c|c|c|c|c|c|c|c|}
\hline problems & 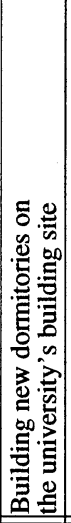 & 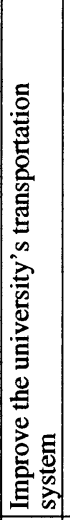 & 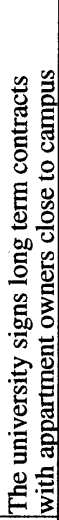 & 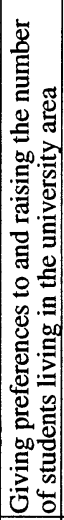 & 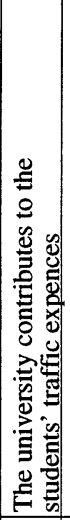 & 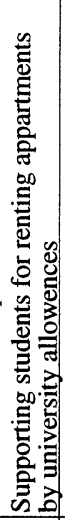 & 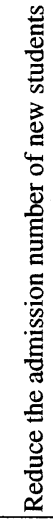 & 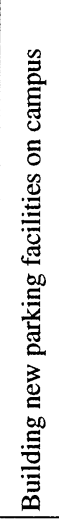 & 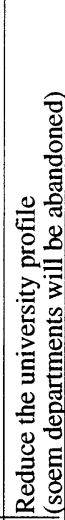 \\
\hline $\begin{array}{l}\text { The number of students living in } \\
\text { dorms close to the campus is limited }\end{array}$ & $\mathrm{x}$ & & $\mathrm{x}$ & $\mathrm{x}$ & $\mathrm{x}$ & & & & $\mathrm{x}$ \\
\hline $\begin{array}{l}\text { Renting an apartment close to the } \\
\text { campus is expensive }\end{array}$ & $\mathrm{x}$ & $\mathrm{x}$ & $\mathrm{x}$ & $\mathrm{x}$ & $\mathrm{x}$ & $\mathrm{x}$ & $\mathrm{x}$ & & $x$ \\
\hline $\begin{array}{l}\text { No new dormitory can be built } \\
\text { on campus }\end{array}$ & $\mathrm{x}$ & $x$ & $\mathrm{x}$ & & & $\mathrm{x}$ & & & $\mathrm{x}$ \\
\hline $\begin{array}{l}\text { The university has a building site } \\
\text { outside the city in driving distance }\end{array}$ & $\mathrm{x}$ & & & & & & $\mathrm{x}$ & $x$ & \\
\hline $\begin{array}{l}\text { The university has resources (annual } \\
\text { budget) for unspecified building activities }\end{array}$ & $\mathbf{x}$ & & & & & & & $x$ & \\
\hline $\begin{array}{l}\text { The university has resources for } \\
\text { supporting a limited number of students }\end{array}$ & & & $\mathrm{x}$ & & $\mathrm{x}$ & $\mathrm{x}$ & $\mathrm{x}$ & & \\
\hline $\begin{array}{l}\text { There are government resources for } \\
\text { building dormitories and parking facilities }\end{array}$ & $\mathrm{x}$ & & & & & & & $x$ & \\
\hline $\begin{array}{l}\text { There are government resources for } \\
\text { unspecified/unrestricted student support }\end{array}$ & & & $\mathrm{x}$ & & $\mathrm{x}$ & $\mathrm{x}$ & $x$ & & $\mathrm{x}$ \\
\hline $\begin{array}{l}\text { The university has not enough } \\
\text { parking facilities }\end{array}$ & $\mathrm{x}$ & $x$ & & $x$ & $\mathrm{x}$ & $x$ & $\mathrm{x}$ & $x$ & $x$ \\
\hline $\begin{array}{l}\text { The university has not enough busses } \\
\text { for transporting students }\end{array}$ & $x$ & $\mathrm{x}$ & & $\mathrm{x}$ & $\mathrm{x}$ & $\mathrm{x}$ & $\mathrm{x}$ & $\mathrm{x}$ & \\
\hline $\begin{array}{l}\text { There is a small number of students } \\
\text { commuting between home and the univ. }\end{array}$ & $\mathbf{x}$ & $\mathrm{x}$ & $x$ & $\mathrm{x}$ & $x$ & & $\mathrm{x}$ & $\mathrm{x}$ & $x$ \\
\hline $\begin{array}{l}\text { The university's teaching capacity } \\
\text { is overloaded }\end{array}$ & $x$ & & $\mathrm{x}$ & $\mathrm{x}$ & $\mathrm{x}$ & $\mathrm{x}$ & $\mathrm{x}$ & $x$ & $x$ \\
\hline
\end{tabular}

Figure 9. The context of the student accommodation problem.

and because of this is a time consuming process, there is an ongoing research effort to generate lattices automatically by computer programs (Wille, 1989). This is important because the concept lattice describing a given task changes with each new data or modification of the context. Frequent - hypothetic or concrete - changes can only be analyzed if one can generate Hasse-diagrams quickly by computers.

Analyzing a Hasse-diagram that represents a design context, means the search for concepts providing satisfactory solutions. Since what 'satisfactory solution' means is subjective, the diagram itself doesn't provide a solution. It supports, however, the decision making process by displaying all the concepts and their order- 


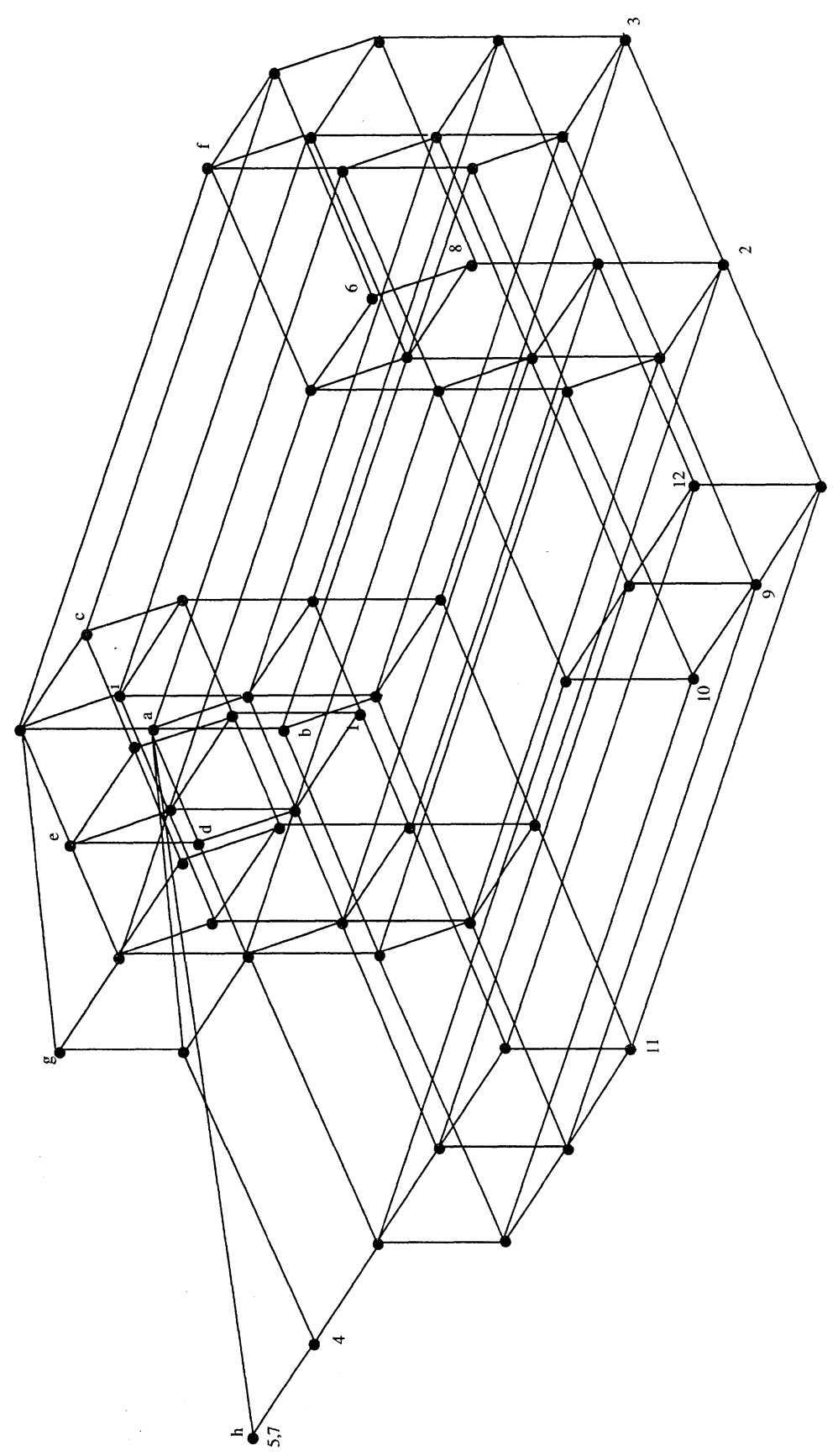

Figure 10. The concept lattice of the student accommodation problem. 
ing. For instance, if we choose a set of decisions of a concept $C_{i}$, let's say $\{a, b, i\}$ then it changes the state of the 'objects' $\{2,3,9,11\}$ of the same concept. In other words, the decisions $a, b$ and $i$ (may) solve the problems 2, 3, 9 and 11. Any set of decisions of a concept $C_{j}$ that is connected by a continuous, steadily downward sloping path to the concept $C_{i}$ solves (or effects) just a (proper) subset of these tasks.

Or we can do the reasoning in the other direction by choosing a set of 'objects' that are essential for solving this particular problem. Similarly, any concept in our lattice containing the chosen objects has a set of decision that will effect these objects. Moreover, any continuous, upward sloping path starting at such a concept leads to concepts with the same property.

In both cases; starting the process with decisions or objects, we might choose a set that does not occur in the lattice. Thus, if we felt that the set $3,9,11$ would have been the key to our context then we would look at the concepts containing these elements. The concept lattice tells us that there is no such set of decisions in our context that effects exactly these three objects and nothing else. The lattice also tells us that the smallest such concept is $A=\{2,3,9,11\}, B=\{a, b, i\}$, and there are exactly five other concepts on up-sloping paths starting at this concept which have the same property.

The next step of the process is to decide which of these six concepts is the most satisfactory. This is a kind of optimization where we have to compare different sets of decisions, or rather their consequences. It is likely that the process involves both: searching for an 'optimal' design state and also for 'optimal' decisions. Optimality means that we want to improve the current situation as much as possible with the minimum amount of effort and sacrifice. It is obvious that design in general is far from being optimizable, and because of the complexity of design problems, it is important that concept lattices (in particular Hasse-diagrams) help us to describe design tasks in exact terms. This description, or model enables us to compare and evaluate decisions. In case of team or collaborative design, concept lattices provide a powerful argumentative tool for discussions and negotiations.

\section{Generating Lattices by Subdirect Products}

Although Hasse diagrams can help us to display and analyze complex design tasks, the generation of Hasse-diagrams becomes increasingly difficult - if not impossible - by the increase of the number of concepts. With increasing number of concepts the diagram becomes large, dense and cluttered and at some point it might fail to provide any help. Not just generating but also 'reading' such diagrams can be difficult. Additionally, not all of the hierarchical structures hidden in the original context are equally significant for the design process. Hasse-diagrams so far do not allow us to make distinctions between different dependencies.

All these problems can be resolved by means of suitable sublattices. Before 
going into the discussion of sublattices, however, let us look at again the Hassediagram in Figure 10. It is not difficult to discover the Boolean sublattices in the diagram having parallel edges. Moreover, we can easily discover that the entire diagram contains two similar looking sublattices (one in the left upper part and the other in the right bottom part of the picture) that are connected by a bundle of parallel lines. By removing these lines the diagram becomes simpler and we still have all the informations of the original diagram. From the simplified diagram in Figure 11 we can obtain the original diagram by moving the 'boxed' diagrams of one sublattice over the diagram of the other sublattice along the connection line of the two boxes. Two points are connected in the original diagram if they cover each other following this translation. Our claim is that we can apply this 'trick' to any concept lattice, and not only we will have a way to represent large lattices efficiently but also we will be able to organize the diagram so that certain structural dependencies become transparent.

As the above described intuitive simplification indicates, sublattices are the key to representation problems. In fact, we are seeking methods to generate and represent lattices from smaller sublattices. There are a couple of methods for generating lattices from appropriate sublattices or decomposing concept lattices into sublattices (Wille, 1983; Wille, 1985). Here we show how lattices can be obtained as the subdirect product of sublattices (Wille, 1987).

Let us first consider contexts having certain structural properties. The aim is to have special cases in which the concept lattice can be generated easily by suitable subcontexts. In other words the idea is to find structural properties of a context which allow us to generate the concept lattice from sublattices. For this purpose, we introduce the following notations: Let us denote a lattice $\mathcal{A}$ by $\left(X, \leq_{\mathcal{A}}\right)$, where $X$ is the underlying set of $\mathcal{A}$ and $\leq_{\mathcal{A}}$ its partial order.

Definition 5.1 The direct product $\mathcal{C}=\mathcal{A} \times \mathcal{B}=\left(X \times Y, \leq_{\mathcal{C}}\right)$ of two lattices $\mathcal{A}=\left(X, \leq_{\mathcal{A}}\right)$ and $\mathcal{B}=\left(X, \leq_{\mathcal{B}}\right)$ is defined such that $\left(x_{1}, y_{1}\right) \leq_{\mathcal{C}}\left(x_{2}, y_{2}\right)$ if and only if $x_{1} \leq_{\mathcal{A}} x_{2}$ and $y_{1} \leq_{\mathcal{B}} y_{2}$. A bounded lattice is a lattice with greatest and smallest element.

Definition 5.2 The horizontal sum $\mathcal{A} \oplus_{h} \mathcal{B}$ of two bounded lattices $\mathcal{A}=\left(X, \leq_{\mathcal{A}}\right)$ and $\mathcal{B}=\left(Y, \leq_{\mathcal{B}}\right)$ is obtained from their cardinal sum $\left(X \dot{\cup} Y, \leq_{\mathcal{A}} \dot{\cup} \leq_{\mathcal{B}}\right)$ (where $U$ denotes disjoint union) by identifying the smallest and the greatest element of the two bounded lattices respectively.

Definition 5.3 The vertical sum $A \oplus_{V} B$ is obtained from their ordinal sum ( $X \dot{\cup} Y, \leq_{\mathcal{A}}$ $\left.\dot{\cup} \leq_{\mathcal{B}} \cup(X \times Y)\right)$ by identifying the greatest element of $\mathcal{A}$ with the smallest element of $\mathcal{B}$.

Definition $5.4 A$ subdirect product $\mathcal{D}=\left(S, \leq_{\mathcal{D}}\right)$ of the lattices $\mathcal{A}=\left(X, \leq_{\mathcal{A}}\right)$ and $\mathcal{B}=\left(X, \leq_{\mathcal{B}}\right)$ is a subset $S \subseteq X \times Y$ closed under the operations $\vee$ and $\wedge$ (sublattice) such that for each element $x \in X$ (or $y \in Y$ ) there is an element $s \in S$ having $x$ (or $y$ ) as its component. 


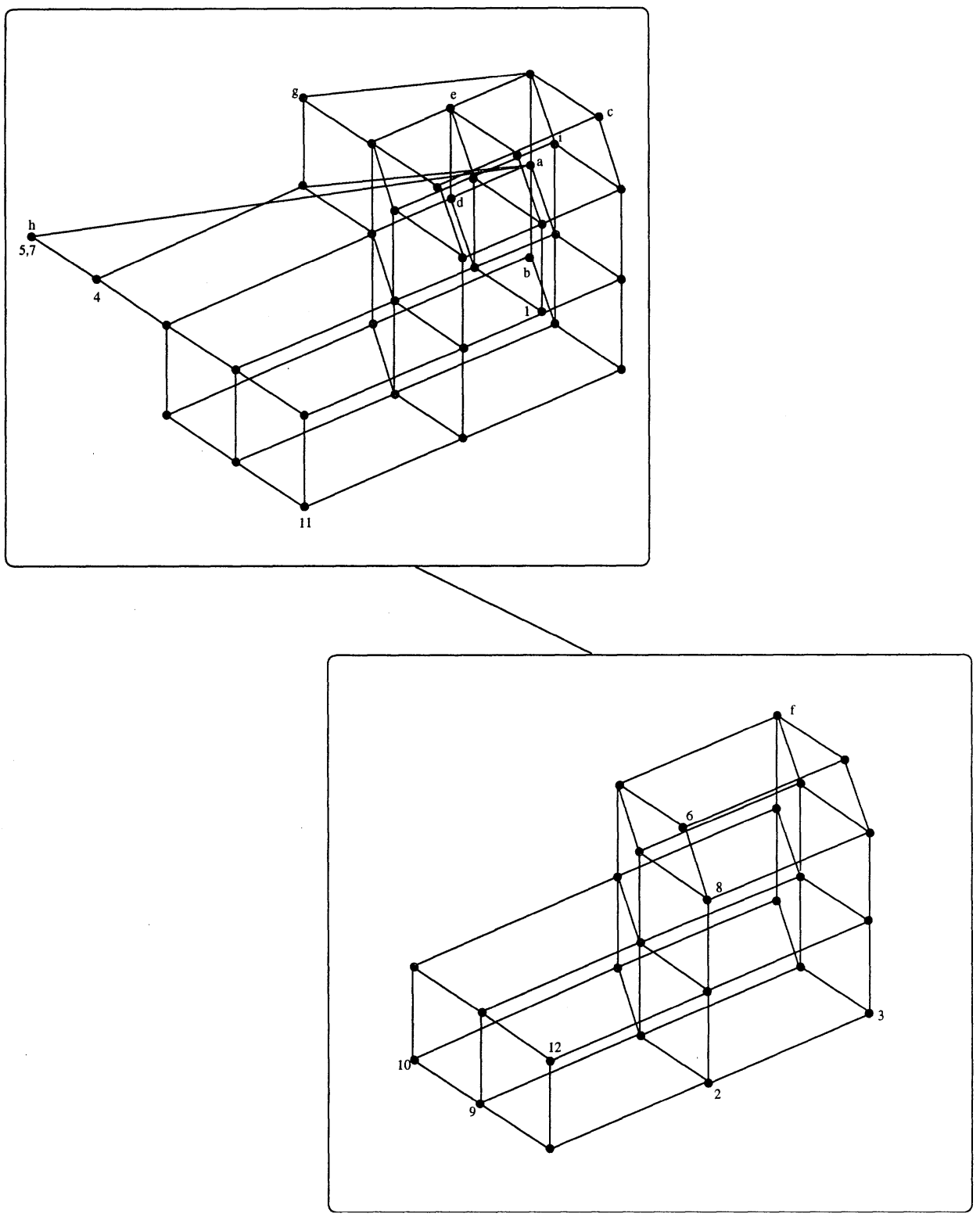

Figure 11. Simplifying the diagram of the lattice describing the student accommodation problem.

The following theorem is applicable in cases where the context contains disjoint subcontexts whose polar sets are empty (Wille, 1987).

Theorem 5.1 Let $C_{1}=\left(X_{1}, Y_{1}, \gamma_{1}\right)$ and $C_{2}=\left(X_{2}, Y_{2}, \gamma_{2}\right)$ be contexts with $X_{1} \cap$ $X_{2}=\emptyset, Y_{1} \cap Y_{2}=\emptyset, X_{1}^{*}=X_{2}^{*}=Y_{1}^{*}=Y_{2}^{*}=\emptyset$ and $\mathcal{L}_{C_{1}}=\mathcal{L}_{1}\left(X_{1}, Y_{1}, \gamma_{1}\right)$ and 
$\mathcal{L}_{C_{2}}=\mathcal{L}_{2}\left(X_{2}, Y_{2}, \gamma_{2}\right)$ the lattices belonging to them.

Then $\mathcal{L}_{C}\left(X_{1} \cap X_{2}, Y_{1} \cap Y_{2}, \gamma_{1} \cap \gamma_{2}\right)$ is isomorphic to the horizontal sum of $\mathcal{L}_{1}$, and $\mathcal{L}_{2}, \mathcal{L}_{C}\left(X_{1} \cap X_{2}, Y_{1} \cap Y_{2}, \gamma_{1} \cap \gamma_{2} \cap X_{1} \cap Y_{2}\right)$ is isomorphic to the vertical sum of $\mathcal{L}_{1}$, and $\mathcal{L}_{2}, \mathcal{L}_{C}\left(X_{1} \cap X_{2}, Y_{1} \cap Y_{2}, \gamma_{1} \cap \gamma_{2} \cap\left(X_{1} \times Y_{2}\right) \cap\left(X_{2} \times Y_{1}\right)\right)$ is isomorphic to the direct product of $\mathcal{L}_{1}$ and $\mathcal{L}_{2}$.

If Theorem 5.1 can not be applied, i.e. we do not have the properties allowing the application of this theorem, then a larger concept lattice can be still generated by using the following general theorem:

Theorem 5.2 Let $C=(X, Y, \gamma)$ be a context, let $\left\{X_{i} \mid i \in I\right\}$ be a partition of $X$ and let $\left\{Y_{j} \mid j \in J\right\}$ be a partition of $Y$. Then $a \vee$-embedding of $\mathcal{L}_{C}(X, Y, \gamma)$ into the direct product of the $\mathcal{L}_{C}\left(X_{i}, Y, \gamma \cap\left(X_{i} \times Y\right)\right)_{i \in I}$ is given by $\langle A, B\rangle \mathcal{P}\langle A \cap$ $\left.X_{i},\left(A \cap X_{i}^{*}\right)_{i \in I}\right\rangle$, a $\wedge$-embedding of $\mathcal{L}_{C}(X, Y, \gamma)$ into the direct product of the $\mathcal{L}_{C}\left(X, Y_{j}, \gamma \cap\left(X \times Y_{j}\right)\right)_{j \in J}$ is given by the mapping $\langle A, B\rangle \mathcal{P}\left\langle B \cap Y_{j},(B \cap\right.$ $\left.\left.Y_{j}^{*}\right)_{j \in J}\right\rangle$.

Conversely, each concept lattice can be represented as a subdirect product of sublattices. We obtain sublattices by dissecting the set $Y$ (or $X$ ) of a context $C=$ $(X, Y, \gamma)$ into subsets $Y_{1}, \cup Y_{2} \cup \ldots \cup Y_{n}=Y$ (or $X_{1}, \cup X_{2} \cup \ldots \cup X_{n}=X$ ) and generating the lattice $\mathcal{L}_{C_{j}}\left(X, Y_{j}, \gamma \cap\left(X \times Y_{j}\right)\right)_{j \in J}\left(\right.$ or $\mathcal{L}_{C_{i}}\left(X_{i}, Y, \gamma\right) \cap\left(X_{i} \times\right.$ $Y)_{i \in I}$ ).

Let us consider the simplest case of a dissection having $Y=Y_{1} \cup Y_{2}$ of the context $C=(X, Y, \gamma)$. In this case we obtain the two concepts $C_{1}=\left(X, Y_{1}, \gamma_{1}\right)$, where $\gamma_{1}=\gamma \cap\left(X \times Y_{1}\right)$ and $C_{2}=\left(X, Y_{2}, \gamma_{2}\right)$ where $\gamma_{2}=\gamma \cap\left(X \times Y_{2}\right)$. From the concepts $C_{1}$ and $C_{2}$ we obtain the two sublattices $\mathcal{L}_{C_{1}}\left(X, Y_{1}, \gamma_{1}\right)$ and $\mathcal{L}_{C_{2}}\left(X, Y_{2}, \gamma_{2}\right)$. The representation of the lattice $\mathcal{L}_{C}(X, Y, \gamma)$ as the subdirect product of $\mathcal{L}_{C_{1}}$ and $\mathcal{L}_{C_{2}}$ goes as follows:

We draw the lattice $\mathcal{L}_{C_{2}}$ as a diagram having 'boxes' (rectangles) instead of points. In each box we draw the Hasse-diagram of the lattice $\mathcal{L}_{C_{1}}$ identically such that moving one box to another along the edges the pictures of $\mathcal{L}_{C_{1}}$ cover each other (i.e. the diagrams are congruent). Let $\{x\}^{*} \cap Y_{1}=A$ and $\{x\}^{*} \cap Y_{2}=B$. Now the concept $\langle A, B\rangle$ of the lattice $\mathcal{L}_{C}$ is in the box $\left\langle B^{*}, B\right\rangle$ of the diagram of $\mathcal{L}_{C_{2}}$ and at the point $\left\langle A^{*}, A\right\rangle$ of the diagram of $\mathcal{L}_{C_{1}}$. Usually, some parts of the diagrams of the lattices $\mathcal{L}_{C_{1}}$ are omitted since not all of the depicted concepts exist. The resulting diagram is a unique representation of $\mathcal{L}_{C}$, i.e. from the 'box diagram' of the lattice one can easily reconstruct the lattice itself. However, it contains less edges than the Hasse-digram of the lattice since 'parallel' and 'complete' edges are replaced by single ones. Parallel edges connect two diagrams in adjacent boxes such that each pair of connected points would cover each other by moving the congruent diagrams. Complete edges connect a single (top or bottom element of the diagram with all the points of a diagram in an adjacency box. (Because of the transitivity of $\leq$ and $\geq$ we need to connect just the smallest or greatest elements in the box.) 


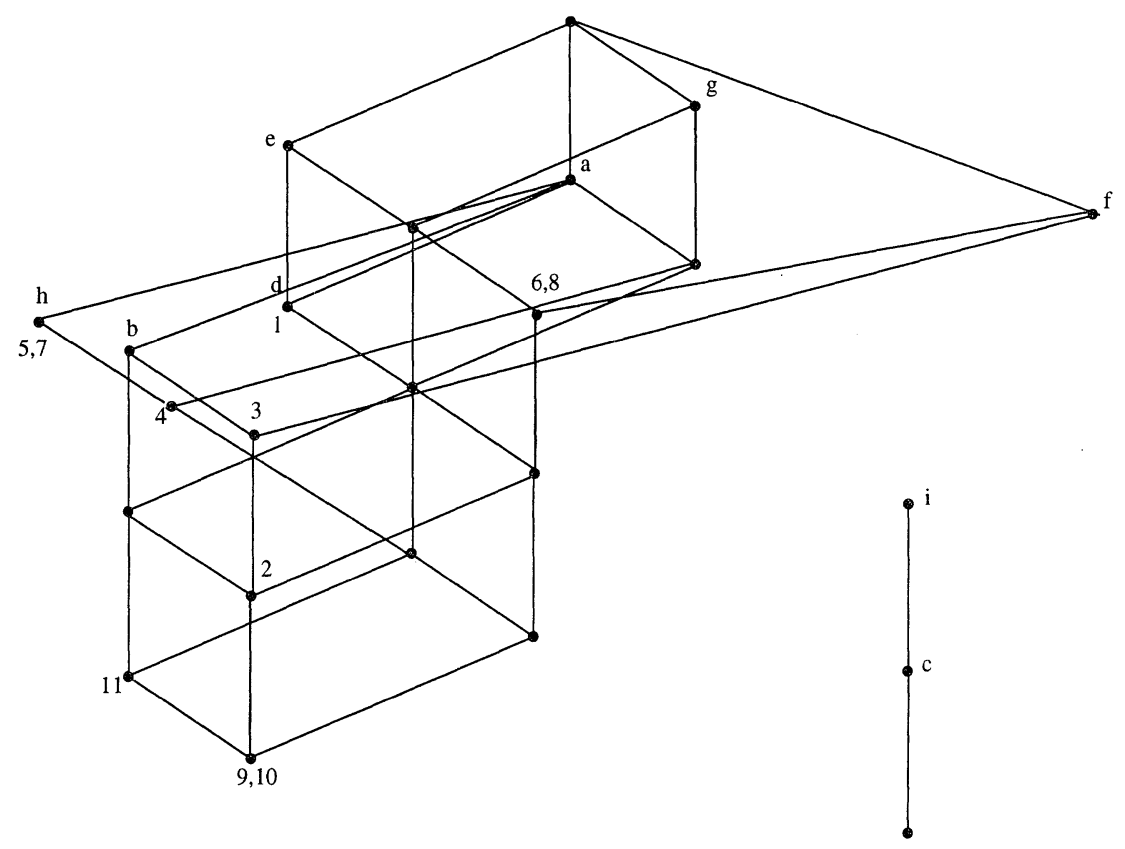

Figure 12. The two sublattices generating the box diagram in Figure 11

The dissection of the set $Y=\{a, b, c, d, e, f, g, h, i\}$ of our example into the subsets $Y_{1}=\{a, b, c, d, e, g, h, i\}$ and $Y_{2}=\{f\}$ provides the two sublattices on Figure 12. The product of these sublattices provides the lattice on Figure 11.

Another dissection of $Y$ into $Y_{1}=\{a, b, c, d, e\}$ and $Y_{2}=\{f, g, h, i\}$ provides interestingly two very similar sublattices. Figure 13 shows both: the two sublattices $\mathcal{L}_{C_{1}}\left(X, Y_{1}, \gamma_{1}\right)$ and $\mathcal{L}_{C_{2}}\left(X, Y_{2}, \gamma_{2}\right)$ and the box diagram of the lattice $\mathcal{L}_{C}(X, Y, \gamma)$.

If the dissection is based on some structural properties then the box diagram displays the order structure according to these structural properties. We could for instance divide the statements about the current planning situation into financial statements $X_{1}=\{2,5,6,7,8\}$ and object descriptive statements $X_{2}=\{1,3,4,9$, $10,11,12\}$. In this case the box diagram would display dependencies that are financial and non-financial nature by having two different representation levels.

By having a decomposition of the set $Y$ (or $X$ ) into three, four, etc. subsets the depth of the structure grows. It becomes a three, four, etc. level box diagram. Even very large concept lattices become representable by using such higher level, nested structures. Besides the fact that nested, multilevel diagrams help us to reduce the number of edges further and make the diagram survey-able, they also provide us more structuring possibilities. In nested diagrams each level represents an aspect we want to emphasize or make transparent. 


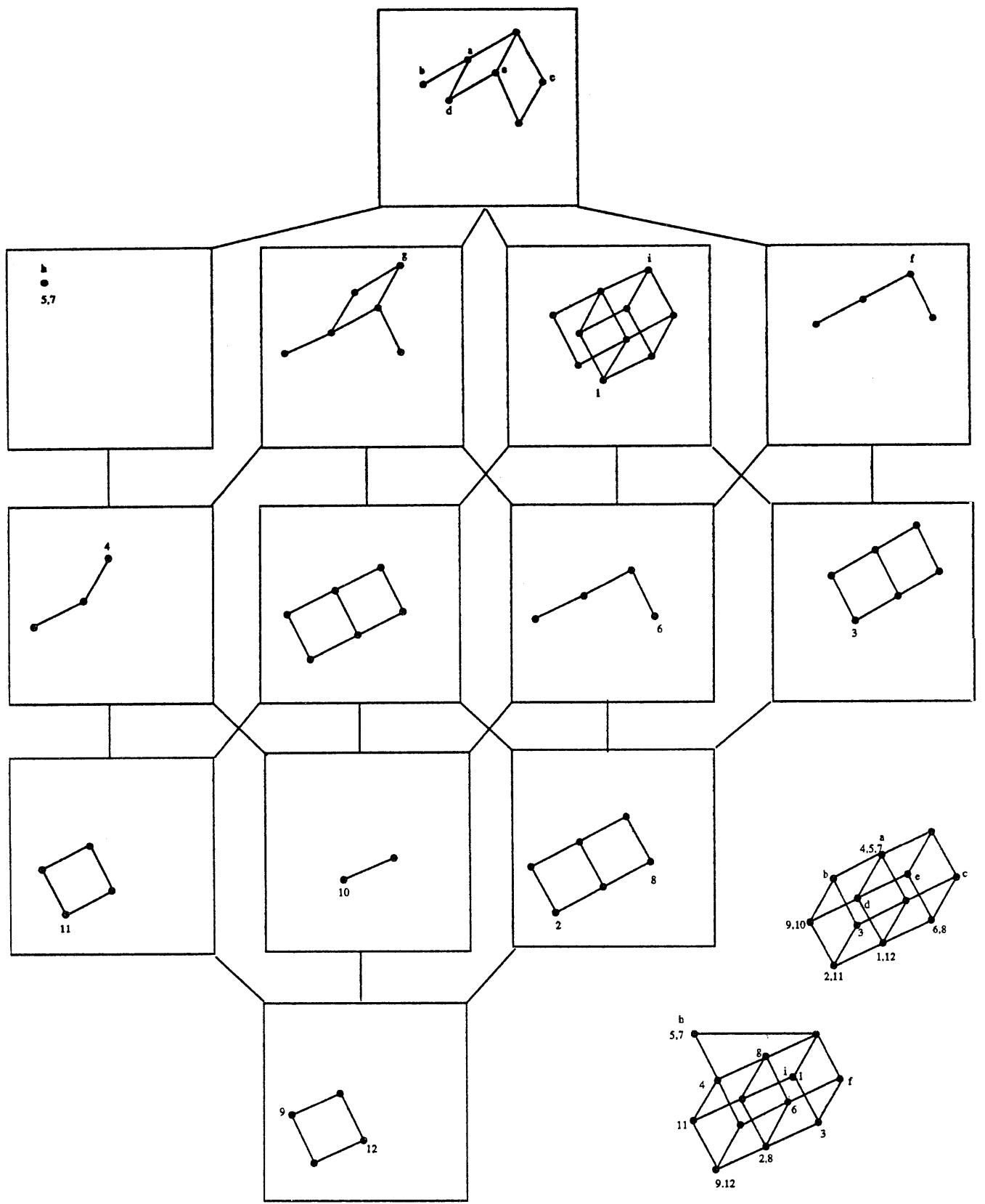

Figure 13. The two sublattice and the box diagram based on the dissection: $Y_{1}=\{a, b, c, d, e\}$, $Y_{2}=\{f, g, h, i\}$ 


\section{Conclusion}

The paper presents the fundamentals of concept analysis and how it can be used for analyzing design tasks. In particular, it shows how to apply Wille's analysis and representation method in design. By using results in lattice theory, we can generate, analyze concept lattices and draw their Hasse-diagrams. Since Hasse-diagrams provide easy access to and easy understanding of concept lattices (even for a layman), the focus of the paper lies in the development and representation of Hassediagrams. Hasse diagrams provide an insight of complex relations that are hidden in the context and usually difficult to obtain otherwise. Since design contexts have not been analyzed so far by these methods, there is a wide range of new applications for concept lattices in the design field.

\section{References}

Atkin, R. H.: 1974, Mathematical Strcture in Human Affairs, Heinemann, London.

Birkhoff, G.: 1967, Lattice Theory (3rd edn), Mathematical Society, Providence, RI.

Gantner, B.: 1987, Algorithmen zur formalen begriffsanalyse, in K. E. Wolf, B. Ganter and R. Wille (eds), Betraege zur Begriffsanalyse, B.I. Wissenschaftsverlag, Mannheim.

Ho, Y-S.: 1982a, The planning process: fundamental issues, Environment and Planning B, 9, 387395.

Ho, Y-S.: 1982b, The planning process: structure of verbal descriptions, Environment and Planning $B, 9,397-420$.

Ho, Y-S: 1982c, The planning process: a formal model, Environment and Planning B, 9, 377-386.

Lenart, M.: 1988, Axiomatic approach to analyzing floor plan topology, 4th International Conference on System Research, Informatics and Cybernetics, Baden-Baden, Germany, pp. 63-71.

Lenart, M.: 1990, Concept lattices as planning models, Design Methods and Theories, 24(1), 11361163.

Macgill, S. M.: 1983, A consideration of Johnson's Q-discrimination analysis, Environment and Planning $B, 9,315-330$.

March, L.: 1982, On Ho's methodological approach to design and planning, Environment and Planning $B, 9,421-427$.

Rival, I.: 1989, Graphical data structures for ordered sets, in I. Rival (ed.), Algorithms and Order, Kluwer, Dordrecht, pp. 3-31.

Wille, R.: 1981, Restructuring lattice theory: An approach based on hierarchies of concepts, in I. Rival (ed.), Proceedings of the Symposium on Ordered Sets, Banff, pp. 445-470.

Wille, R.: 1983, Subdirect decomposition of concept lattices, Algebra Universalis, 17(3), 275-283.

Wille, R: 1984, Line diagrams of concept systems, International Classification, 11(2), 77-86.

Wille, R.: 1985, Tensorial decomposition of concept lattices, Order, 2, 81-95.

Wille, R.: 1987, Subdirect product construction of concept lattices, Discrete Mathematics, 63, 305313.

Wille, R.: 1989, Lattices in data analysis: How to draw them with a computer, in I. Rival (ed.), Algorithms and Order, Kluwer, Dordrecht, pp. 33-58.

Wille, R.: 1992, Concept lattices and conceptual knowledge systems, Computers Math. Applic., 23(6-9), 493-515. 